Classification

Physics Abstracts

$05.40-61.40 \mathrm{~K}-66.90-82.90$

\title{
Quasielastic light scattering by semi-dilute solutions of macromolecules at theta temperature
}

\author{
M. Adam and M. Delsanti \\ Laboratoire Léon Brillouin, CEN Saclay $\left({ }^{*}\right), 91191$ Gif-sur-Yvette Cedex, France
}

(Reçu le 14 octobre 1983, révisé le 9 janvier 1984, accepté le 17 janvier 1984)

Résumé. - La lumière diffusée par des solutions semi-diluées de polystyrène cyclohexane au point théta, est analysée à l'aide d'un spectromètre hétérodyne.

A partir de la fonction de corrélation de l'intensité qui est non exponentielle on détermine deux temps caractéristiques :

- un temps court, dont la dépendance avec le vecteur de transfert $q$ sera discutée.

- un temps long, indépendant de $q$, dont la valeur correspond au temps de relaxation viscoélastique le plus long.

\footnotetext{
Abstract. - We analyse the light scattered by semi-dilute polystyrene cyclohexane solutions at theta temperature, using a heterodyne spectrometer.

In the non exponential intensity correlation function one identifies two characteristic times :

- a short one which is dependent of the momentum transfer $q$

- a long one, $q$ independent, which corresponds to the longest viscoelastic relaxation time.
}

The correlation function of the light scattered by a semi-dilute solution of polystyrene in cyclohexane at theta temperature $\left(35^{\circ}\right)$ has a line shape which is strongly non exponential [1]. Then :

- we use a heterodyne technique [2]

- we fit the experimental points to an exponential line shape :

$$
\langle I(t) I(0)\rangle=A \exp (t / \tau)+B,
$$

where $A, \tau$ and $B$ are determined using a three-parameter fitting procedure. For each spectrum, we calculate :

- the quality factor $Q$, which allows us to evaluate the distortion of an experimental curve from an exponential function; $Q$ is defined by :

$$
Q=1-\frac{\left|\sum_{i=1}^{N-1} \varepsilon_{i} \varepsilon_{i+1}\right|}{\sum_{i=1}^{N} \varepsilon_{i}^{2}},
$$

(*) Laboratoire commun CEA, CNRS. 
where $N$ is the number of experimental points $(128)$ and $\varepsilon_{i}$ the deviation of the $i$ th experimental from the $i$ th theoretical point.

- the relative standard deviation $V$ of $\tau$ :

$$
V^{2}=\frac{\left\langle\delta \tau^{2}\right\rangle}{\tau^{2}} \propto \frac{1}{N-3} \Sigma_{i} \varepsilon_{i}^{2}
$$

- the ratio $Q / V \times 100$ which allows us to compare the distortion of experimental curves, even if they have different $V$ values.

The continuous spectrum corresponding to $\theta$ conditions treated using this procedure allows us to find a characteristic $\tau$ whatever is the preset time per channel $\Delta t$ of the correlator. Those characteristic times are bounded by two times : the longer one $\tau_{\mathrm{L}}$ and the shorter one $\tau_{\mathrm{s}}$. Indeed if $\Delta t \geqslant \frac{\tau_{\mathrm{L}}}{N}$ one observes a base line and if $\Delta t<\frac{\tau_{\mathrm{s}}}{N}$ one observes a decreasing straight line. This behaviour is illustrated by figure 1 where the characteristic time $\tau$ obtained for different scattering angles is plotted against $\Delta t$. From this figure, it turns out that one long time plateau $\tau_{\mathrm{L}}$ is observed independent of the momentum transfer $q$ :

$$
q=\frac{4 \pi}{\lambda} \sin \frac{\theta}{2}
$$

where $\lambda$ is the wavelength of the incident light in cyclohexane and $\theta$ the scattering angle.

The short time $\tau_{\mathrm{s}}$ of figure 1 , corresponding to a maximum value of the ratio $Q / V \times 100(\approx 0.5)$, is $q$ dependent. One must remark that the ratio $Q / V \times 100$ increases with temperature and becomes larger than 1 around $65^{\circ} \mathrm{C}$ and simultaneously, the amplitude of the spectrum corresponding to the long time plateau of figure 1 decreases and becomes too small to be detectable around $65^{\circ} \mathrm{C}$. The same effects are observed at $\theta$ temperature at a $q$ vector such that $\tau_{\mathrm{L}}<\tau_{\mathrm{s}}$.

The longest characteristic time $\tau_{\mathrm{L}}$ measured by quasielastic light scattering is of the same order of magnitude as the longest viscoelastic relaxation time $T_{\mathrm{R}}$ measured for the same solutions using the magnetorheometer [3] (see Fig. 1 and table I).

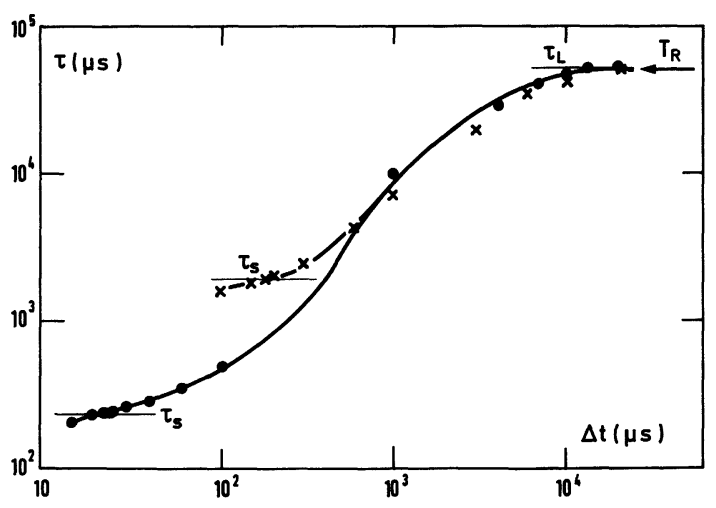

Fig. 1. - Variation of the characteristic time $\tau$ of the spectrum as a function of the time per channel of the correlator $\Delta t\left(\log \log\right.$ scale) $\times \sin \frac{\theta}{2}=0.213, O \sin \frac{\theta}{2}=0.525$, the sample characteristics are : molecular weight $M_{\mathrm{w}}=3.84 \times 10^{6}$, and concentration $C=5.02 \times 10^{-2}(\mathrm{~g} / \mathrm{g})$. The arrow indicates the longest viscoelastic relaxation time $T_{\mathrm{R}}$ and full lines are a guide for the eye. 
Table I. - Values of longest relaxation times measured by, $T_{\mathbf{R}}$ magnetorheometer, $\tau_{\mathbf{L}}$ light scattering experiments. Characteristics of the samples used.

\begin{tabular}{|c|c|c|c|}
\hline$M_{\mathrm{W}} \times 10^{-6}$ & $C \times 10^{2}(\mathrm{~g} / \mathrm{g})$ & $T_{\mathrm{R}}(\mathrm{s})$ & $\tau_{\mathrm{L}}(\mathrm{s} ;$ \\
\hline \multirow{2}{*}{6.77} & 5.01 & $0.30 \pm 0.03$ & $0.43 \pm 0.1$ \\
& 3.24 & $0.082 \pm 0.008$ & $0.11 \pm 0.01$ \\
3.84 & 5.02 & $0.044 \pm 0.004$ & $0.05 \pm 0.01$ \\
& 6.65 & $0.097 \pm 0.01$ & $0.088 \pm 0.01$ \\
20.6 & 10.05 & $0.312 \pm 0.03$ & $0.55 \pm 0.1$ \\
& 2.38 & $2.98 \pm 0.3$ & $2.12 \pm 0.4$ \\
\hline
\end{tabular}

In a semi-dilute theta solution the reduced length is $q \xi$, where $\xi$ is the concentration fluctuations correlation length. Experimentally, using the numerical values of $\xi$ determined by neutron scattering [4] we find that :

- when $q \xi>\frac{1}{3}$, the inverse of the shortest relaxation time $\tau_{\mathrm{s}}^{-1}$ varies faster than $q^{2}$ (Fig. 2). This last point is in contrast with one conclusion of reference [5] which predicts, in this condition, a $q$ dependence;

- when $q \xi<\frac{1}{3}$, the inverse of the shortest characteristic time is $q^{2}$ dependent (Fig. 3). It is then possible to extract a diffusion coefficient.

At the present time the problem to be solved is the dependence on concentration and molecular weight of the cooperative diffusion coefficient deduced from the short characteristic time. However, the important result of this letter is that the longest relaxation time, here measured, is comparable to the largest viscoelastic time. This is in agreement with the theoretical prediction of reference [5].

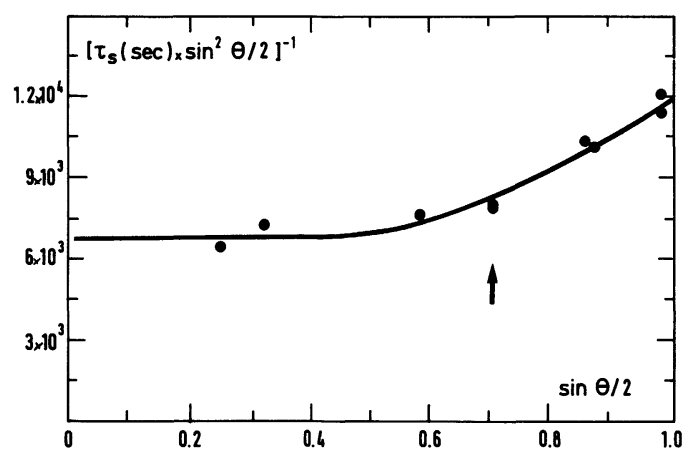

Fig. 2. - Variation of the inverse characteristic time $\tau_{\mathrm{s}}^{-1}$ divided by $\sin ^{2}\left(\frac{\theta}{2}\right)$, as a function of $\sin \frac{\theta}{2}$. The arrow indicates the value of $\sin \frac{\theta}{2}$ for which $q \xi=\frac{1}{3}$ and full line is a guide for the eye. The sample characteristics are : $M_{\mathrm{w}}=6.77 \times 10^{6}, C=3.24 \times 10^{-2}(\mathrm{~g} / \mathrm{g})$. 


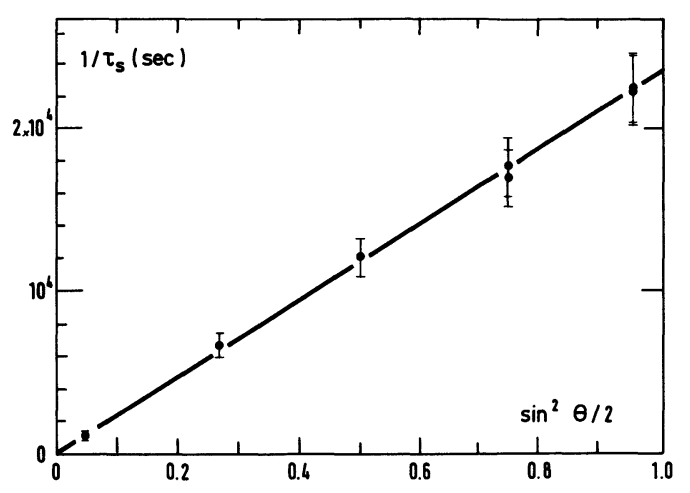

Fig. 3. - Example of variation of the inverse of the characteristic time $\tau_{\mathrm{s}}^{-1}$ as a function of $\sin ^{2} \frac{\theta}{2}$ with $q \xi<\frac{1}{3}$. The sample characteristics are $: M_{\mathrm{w}}=20.6 \times 10^{6}, C=0.10(\mathrm{~g} / \mathrm{g})$.

\section{References}

[1] See for example, Munch, J. P., Hild, G., Candau, S., Macromolecules 16 (1983) 71.

Amis, E. J., Han, C. C., Matsushita, Y., preprint.

NoSE, T., ChU, B., Macromolecules 12 (1979) 590.

[2] Adam, M., Delsanti, M., Macromolecules 10 (1977) 1229.

[3] Adam, M., Delsanti, M., Pieranski, P., Meyer, R., to appear in Revue Phys. Appl. (March 84). Adam, M., Delsanti, M., to be published.

[4] Cotton, J. P., Nierlich, M., BouÉ, F., Daoud, M., Farnoux, B., Jannink, G., Duplessix, R., Picot, C., J. Chem. Phys. 65 (1976) 1101.

[5] Brochard, F., J. Physique 44 (1983) 39. 